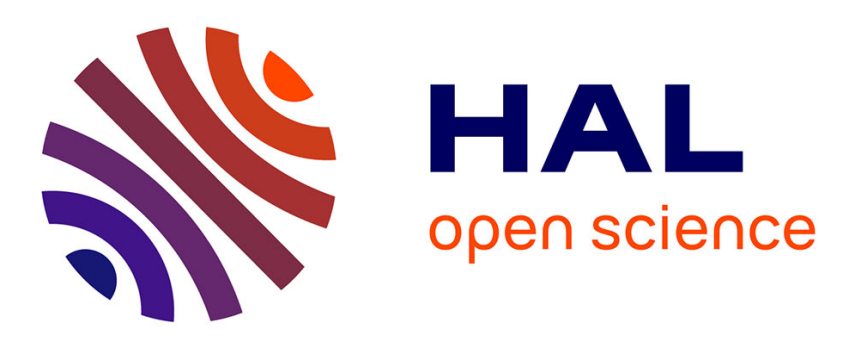

\title{
Bipolar Conducting Polymer Crawlers Based on Triple Symmetry Breaking
}

Bhavana Gupta, Bertrand Goudeau, Patrick Garrigue, Alexander Kuhn

\section{To cite this version:}

Bhavana Gupta, Bertrand Goudeau, Patrick Garrigue, Alexander Kuhn. Bipolar Conducting Polymer Crawlers Based on Triple Symmetry Breaking. Advanced Functional Materials, 2018, 28 (25), pp.1705825. 10.1002/adfm.201705825 . hal-02101540

\section{HAL Id: hal-02101540 \\ https://hal.science/hal-02101540}

Submitted on 25 Apr 2019

HAL is a multi-disciplinary open access archive for the deposit and dissemination of scientific research documents, whether they are published or not. The documents may come from teaching and research institutions in France or abroad, or from public or private research centers.
L'archive ouverte pluridisciplinaire HAL, est destinée au dépôt et à la diffusion de documents scientifiques de niveau recherche, publiés ou non, émanant des établissements d'enseignement et de recherche français ou étrangers, des laboratoires publics ou privés. 


\section{Bipolar conducting polymer crawlers based on triple symmetry breaking}

Bhavana Gupta, Bertrand Goudeau, Patrick Garrigue, Alexander Kuhn*

Univ. Bordeaux, ISM UMR CNRS 5255, Bordeaux INP, ENSCBP, 16 avenue Pey Berland, 33607 Pessac, France

E-mail: kuhn@enscbp.fr

Keywords: Bipolar electrochemistry, conducting polymers, actuation, artificial muscle, robotics

Bipolar electrochemistry can be used in different ways to induce motion of an object, for example by generating gas bubbles in an asymmetric way or by a wireless self-regeneration mechanism due to the intrinsic symmetry breaking of this concept. Here we explore a complementary approach based on conducting polymer objects addressed in solution by an electric field. The presence of the latter results in a differential polarization of the polymer, thus enabling its oxidation at one extremity and its reduction at the opposite side. This triggers different degrees of swelling and shrinking, leading to important deformations of the object. Combined with an additional asymmetry in the polymer surface morphology, a periodic switching of the electric field orientation allows exploiting these deformations to induce directed crawling motion. This first example of a wireless biomimetic crawler based on conducting polymers opens interesting long-term perspectives in several areas such as for example wireless valves, pumps and (micro)robotics.

\section{Introduction}

In recent years many efforts have been devoted by the scientific community to explore new mechanisms allowing objects to transform energy from the surrounding medium into motion. Prominent examples are micromotors or microswimmers. ${ }^{[1]}$ The common point of all these approaches is that symmetry needs to be broken in one way or the other in order to generate directed motion, ${ }^{[2-6]}$ thus leading to systems which can be used for various applications ranging from biosensing and drug delivery to environmental remediation. ${ }^{[7-10]}$ In many of 
these examples locally modified electrokinetics are responsible for the motion, ${ }^{[6,11-14]}$ but also other energy sources such as light or magnetic fields have been studied in this context. ${ }^{[15-17]}$ One concept which has emerged as an efficient approach to break the symmetry of chemical systems in a straightforward way, and thus to induce either directly or indirectly motion, is bipolar electrochemistry (BPE) ${ }^{[13]}$ With BPE, redox reactions can be performed in a wireless way on the surface of conducting or semi-conducting objects when they are placed in an electric field between two feeder electrodes. ${ }^{[18-21]}$ We've recently proposed BPE to actuate conducting polymers in a wireless way. ${ }^{[22]}$ In this case actuation is due to a differential change in volume as a consequence of ion movement in and out of the polymer upon its localized oxidation and reduction. This leads to an artificial muscle type behavior, which has already been explored previously with various conducting polymers such as polypyrrole (PPy), polyaniline (PANI) and poly (3, 4 dioxythiophene) (PEDOT) by classic electrochemical addressing. ${ }^{[23-26]}$ Since such polymeric films can be obtained as freestanding films, different types of devices have been elaborated, able to move in specific directions. ${ }^{[27-29]}$ However, all these devices require a direct connection to an external power supply. BPE is able to circumvent this problem because electrochemical reactions can be carried out on an object without the need of a physical connection to a power supply. In this contribution we suggest to use electrochemistry as the basis for a triple breaking of symmetry:

-morphological asymmetry generation during the synthesis of a freestanding polymer film -polarization asymmetry due to the presence of an electric field -mechanical strength asymmetry obtained via differential post-synthesis reactions In fine, the combination of these three different types of asymmetry allows a conducting polymer object to perform directed crawling motion. 


\section{Results and discussion}

In order to achieve efficient directed crawling of the conducting polymer object, it is crucial to break the symmetry of the system in three distinct ways. The first type of introduced asymmetry concerns the morphology of the film ensuring its inhomogeneous response to the surrounding environment. ${ }^{[29,30]}$ Thus, we first prepared robust polypyrrole films of different thickness for which the surface morphology at the two faces is different. The film surface which is facing the electrolyte during the synthesis is found to be rough and porous, whereas the opposite side (in contact with the gold substrate) is smooth and compact as shown in Figure 1a and $\mathrm{b}$ respectively. As the movement of charge compensating ions into and out of the polymer film strongly depends on the roughness of the polymer, the rough face is more appropriate for ion exchange than the smooth one. Therefore, the as-synthesized film is a good and intrinsic analogue of a classic bilayer film, often used for actuation.

The second symmetry break is related to the unequal incorporation of counterions through the two interfaces when bipolar reduction and oxidation occurs at the two extremities of the object (Figure 1c). The oxidized form of the polymer, as it is obtained during the synthesis without any post-treatment, is rather stiff due to the inter-chain interactions or cross-linking due to the anions immobilized in the polymer matrix. Reduction of the conducting polymer causes either the removal of anions from the polymer or an insertion of cationic species into the polymer matrix in order to maintain charge neutrality. Which pathway is used for charge compensation depends on the size of the anion that is present during the polymerization. If the anions are small, usually they will leave the polymer during reduction and get reintegrated during the oxidation. Thus, reduction leads to shrinkage and oxidation results in swelling. However, for large anions, like in the present case where we employ bulky dodecyl benzene sulfonate, an opposite effect is encountered. During reduction, cations move into the polymer and induce a volume increase (Scheme 1), whereas upon oxidation cations are released and cause shrinking. ${ }^{[18,19]}$ 

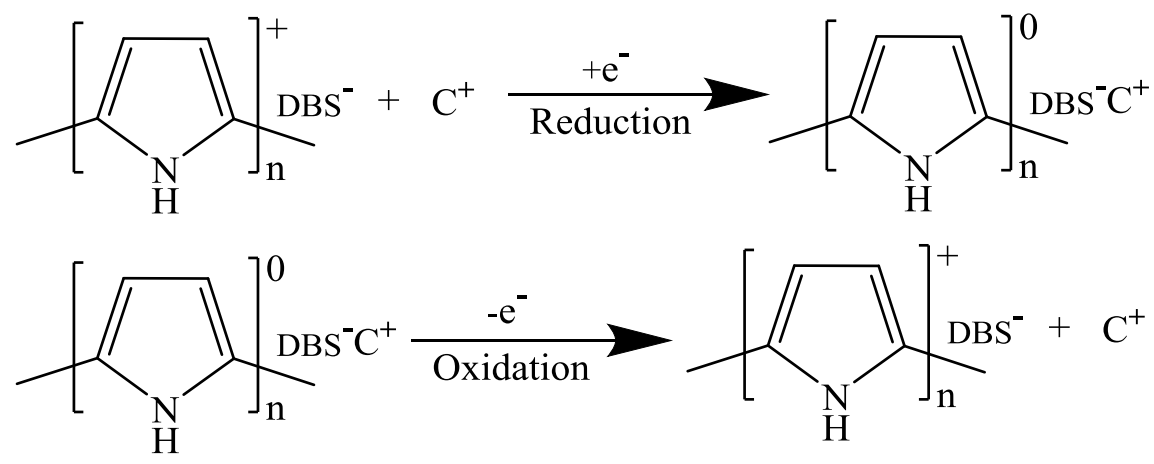

Scheme 1 Electroactivity of polypyrrole during reduction (top line) and oxidation (bottom line)

Cations are crossing the polymer/electrolyte interface preferentially at the rough face of the polymer strip due to the significantly higher active surface area. Thus, the rough face tends to swell and the polymer strip is bending upwards (Figure 1d, left side). In contrast to that, the part of the polymer which is facing the negative feeder electrode is undergoing an oxidation reaction. Therefore, cations are expelled through the rough face due to its higher porosity, thus triggering a more pronounced shrinking. Consequently, the polymer strip tends to bend downwards (Figure 1d, right side). 


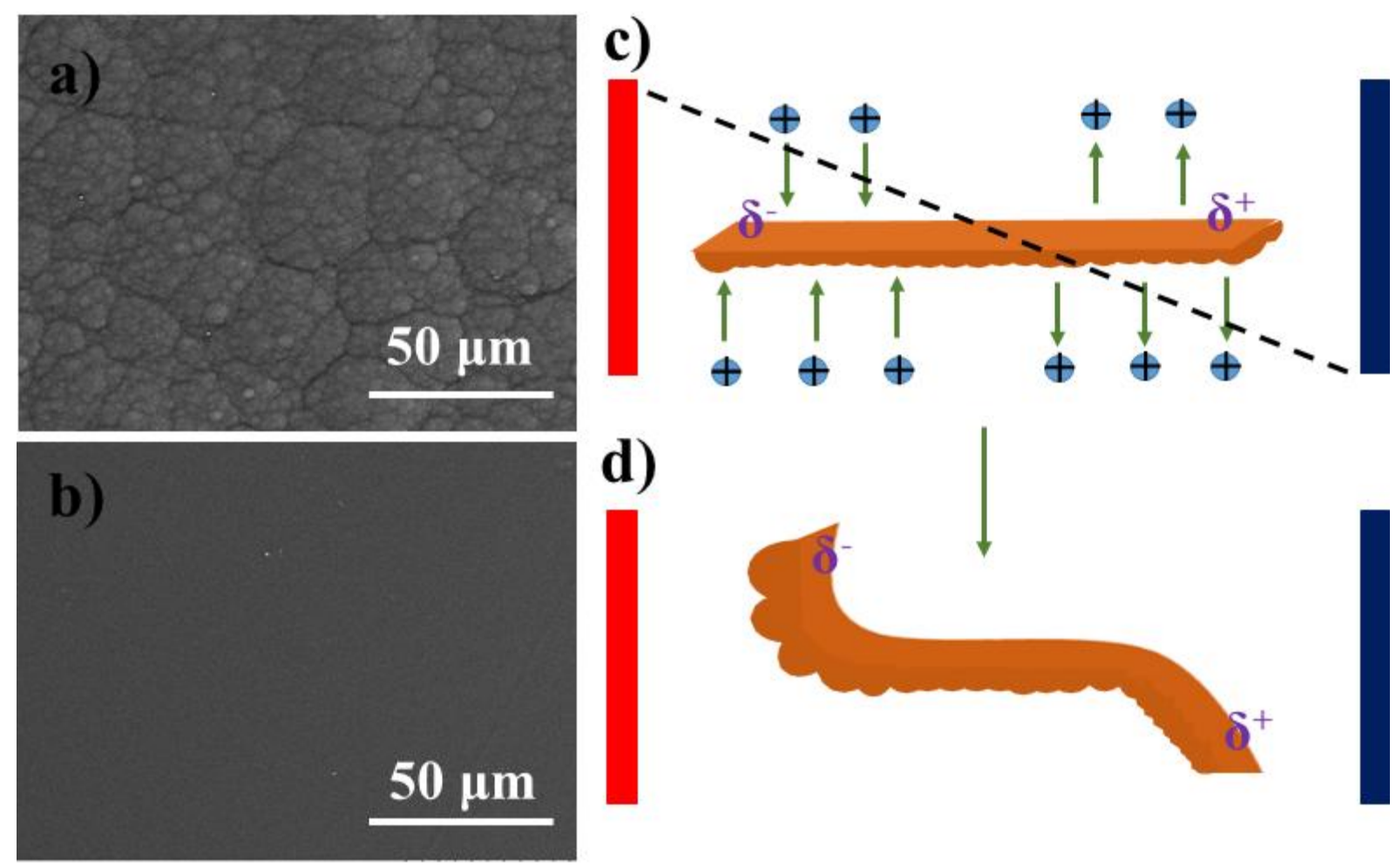

Figure 1 a) Top face (facing the electrolyte during polymerization), and b) bottom face (facing the substrate during polymerization) of an as-synthesized polypyrrole film c) schematic side view of ion movement across a partially oxidized polypyrrole/electrolyte interface in the presence of parallel electric field (flat face oriented up and rough face oriented down) and d) bending after charge compensating ion transport due to differential swelling and shrinking. Red: positive feeder electrode; Blue: negative feeder electrode. $\delta^{+}$and $\delta^{-}$indicate the polarization of polypyrrole film with respect to the solution. The dotted line represents a first order approximation of the potential gradient in the bipolar cell, which in reality is not linear close to the feeder electrodes and around the bipolar object.

Introduction of a third type of asymmetry is possible if the conducting polymer is already completely oxidized before the bipolar experiment. In this case, further oxidation at the positively polarized extremity during the first polarization step will lead to over-oxidation and a partial degradation of the polymer at this location. The polymer becomes less electroactive and less suitable for ion exchange at this extremity, thus leading to weaker bending. To 
confirm this third break of symmetry between the two extremities, scanning electron microscopy images of the polymer strip have been recorded after the bipolar experiment (Figure 2). The end of the polymer strip which was facing the negative feeder electrode during the first bipolar cycle shows an extremely different morphology compared to the part of the polymer which was facing the feeder anode. This asymmetry can even be seen at a macroscopic scale (Fig 2a).

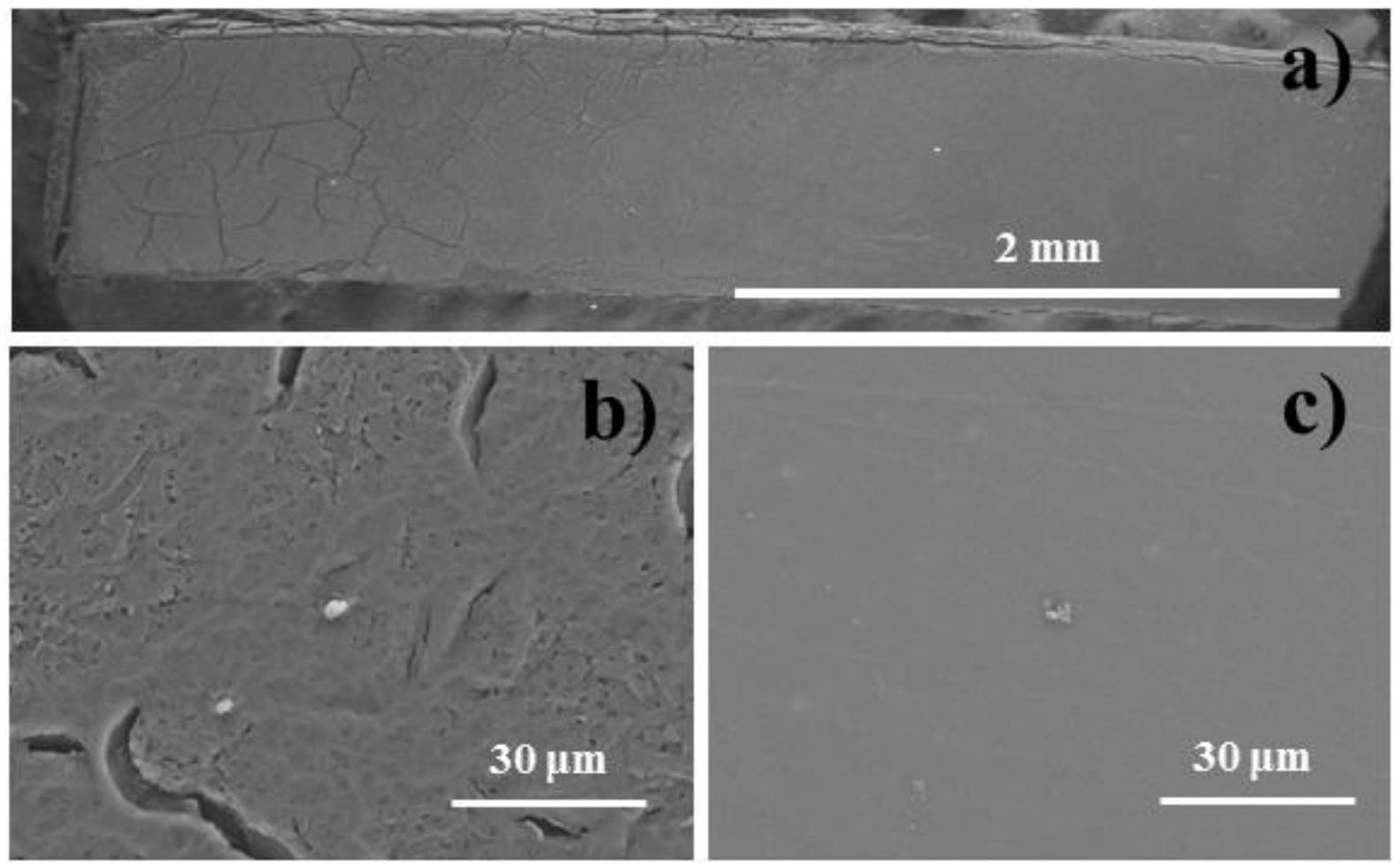

Figure 2 a) Low magnification SEM image of the polymer strip after bipolar switching at 20 $\mathrm{V}$ in $0.5 \mathrm{M} \mathrm{LiClO}_{4}$; the left polypyrrole side experienced an overoxidation during the first bipolar cycle and the right side underwent a reduction during the first bipolar cycle; microscopic image of polypyrrole b) at the left and c) right extremity.

The polymer extremity participating in the first oxidation reaction shows cracks on the surface (Figure 2b), and their density gradually decreases when going towards the extremity that participated in the reduction reaction. The latter one has almost the same smooth microscopic morphology than the as-synthesized polymer support (Figure 2c), indicating the intrinsic 
stability of the polymer even after many switching cycles. This additional third asymmetry is a crucial ingredient for the directed motion of the polypyrrole film, because it allows inhomogeneous bending and relaxation.

Most importantly, the bipolar actuation is switchable. After changing the polarity of the feeder electrodes, the direction of polymer bending is reversed. This feature is extremely important for directional motion, however reversibility alone is not enough as it would lead only to a stationary periodic actuation. As mentioned above, the additional requirement for inducing net motion in a specific direction is that the bending of the two extremities during the bipolar electrochemical process is unequal. In the case of an initially fully oxidized polymer, the over-oxidation of the polypyrrole extremity facing the negative feeder electrode during the first polarization cycle introduces this additional asymmetry. The polymer at this extremity undergoes a partial degradation (vide supra), which makes it stiffer and therefore distinguishable from the opposite extremity. At the same time the effective length of the polymer object which is experiencing the electric field is decreased during this first cycle due to the over-oxidized section. Thus, for the subsequent cycles the overall polarisation of the object is less and no over-oxidation occurs at the opposite extremity when this one gets positively polarized. The resulting unequal mechanical properties of the two ends of the polymer strip enable now a net motion in the direction of the polymer extremity which was initially facing the positive feeder electrode, because this end shows stronger bending compared to the over-oxidized end which is stiffer. This mechanism is schematically illustrated in Figure 3. 

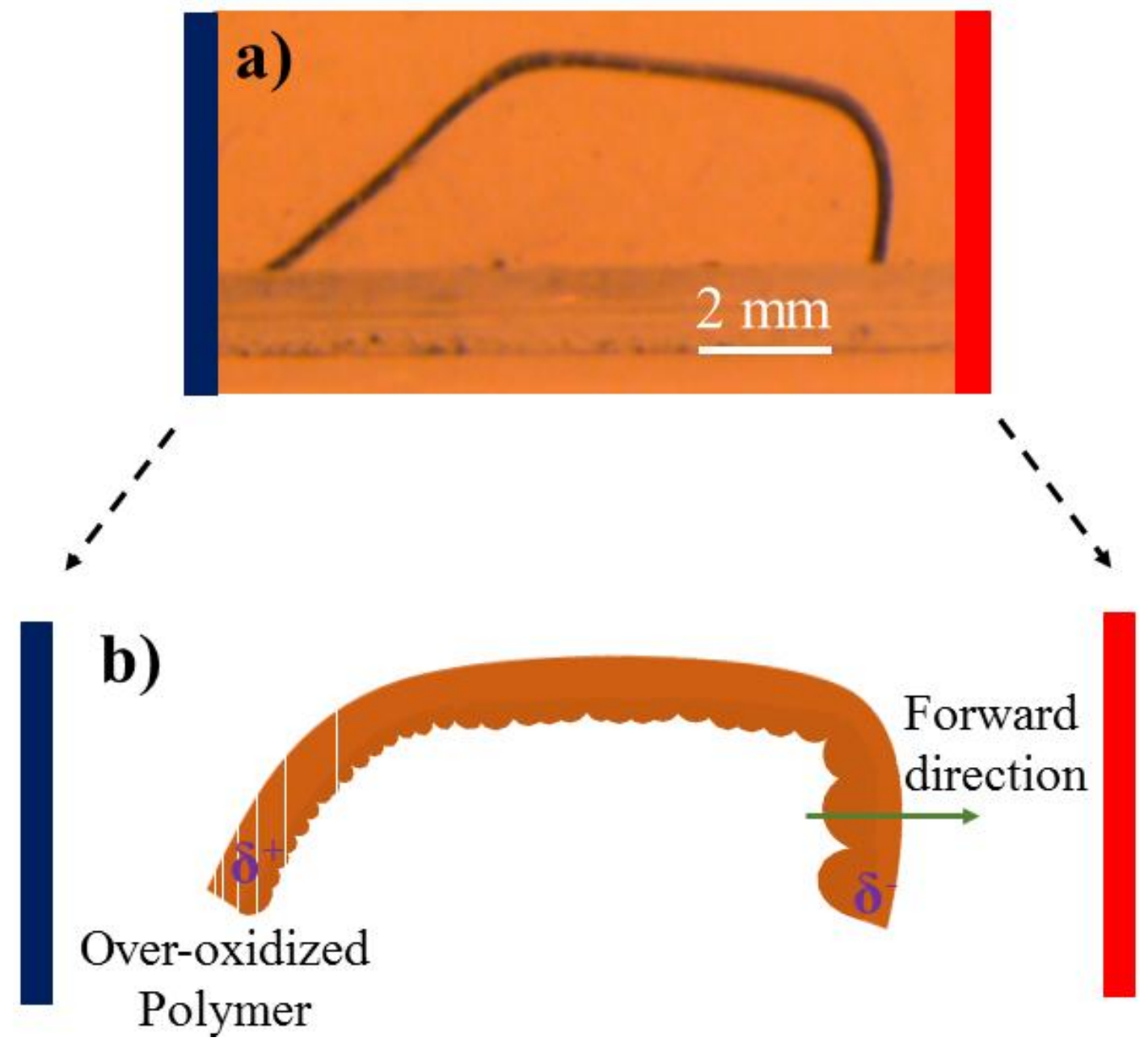

Figure 3 a) Asymmetric shape of the polypyrrole strip during potential switching. b) Schematic illustration of the asymmetric shape of the two ends of the polymer responsible for the crawling in a specific direction in the presence of $0.5 \mathrm{M} \mathrm{LiClO}_{4}$ and at $20 \mathrm{~V}$. Red: positive feeder electrode; blue: negative feeder electrode. $\delta^{+}$and $\delta^{-}$indicate polarization of polypyrrole film with respect to the solution

An as-synthesized fully oxidized polymer strip $(10 \mathrm{x} 1 \mathrm{~mm})$ is positioned in the centre of the bipolar electrochemical cell at the bottom in such a way that the smooth surface is facing upwards and the rough surface downwards. A potential difference of $20 \mathrm{~V}$ is applied between the graphite feeder electrodes placed $5 \mathrm{~cm}$ apart from each other. The maximum potential 
difference $\Delta V$ that might be experienced by the two extremities of the polymer strip can be extracted from the following equation:

$$
E=\frac{\Delta V}{l} L
$$

with $E$ being the potential difference between the feeder electrodes, $L$ the distance between the feeder electrodes $(5 \mathrm{~cm})$ and $l$ the length of the polymer strip $(1 \mathrm{~cm})$. This leads to a theoretical potential difference of $4 \mathrm{~V}$ between the two ends of the polymer. However, there are various sources of potential loss in the bipolar cell such the resistance created by the Nafion membranes, which are separating the electrode compartments, and an important potential drop at the feeder electrodes. In order to estimate the real potential drop along the polymer object, electrical connections have been attached at its two extremities and their potential difference was measured while applying $20 \mathrm{~V}$ to the feeder electrodes. A typical $\Delta V$ value of 1.4-1.6 V was measured in this case. These values are high enough to allow the simultaneous reduction and oxidation of polypyrrole when comparing with a typical cyclic voltammogram of such a polymer film. The extremity of the strip which is facing the positive feeder electrode participates in the reduction reaction, leading to the insertion of cations during the first bipolar cycle. When switching the polarity after $5 \mathrm{~s}$, the polymer extremity, which previously underwent the reduction reaction, is now oxidized, accompanied by the expulsion of cations. The swelling, which was a result of the reduction reaction in the previous cycle, allows a faster expulsion of the cations during oxidation of this extremity. Hence, the downward bending occurs faster. The previously over-oxidized polymer extremity now undergoes a reduction reaction, but an overall upward bending is not observed due the over-oxidized state of the polymer which introduces irreversible morphological and mechanical changes (vide supra). 
In conventional electrochemistry the amplitude of bending depends on thickness. The thickness of the polymer should not be too high because otherwise it resists to bending due to its stiffness, but should also not be too low because otherwise the ion movement is not sufficient to generate enough volume change for efficient bending. ${ }^{[23,31]}$ Therefore, it is crucial for obtaining a bipolar crawler to optimize the thickness of the polymer. In order to illustrate the importance of this parameter we synthesized polymer films with different thickness and studied their dynamic behavior. As an example, we compare here two films which have been obtained after the same reaction time ( 2 hours), but at different potentials i.e. 0.7 and $0.8 \mathrm{~V}$. Their thickness is 60 and $85 \mu \mathrm{m}$, respectively. The bending of the latter one is less pronounced (Figure 4b) compared to the first one (Figure 4e). In addition to the difference in thickness, also another parameter might be influencing the difference in bending amplitude. The polymerization kinetics is slower in the case of $0.7, \mathrm{~V}$ which leads to the formation of shorter polymer chains with a smaller degree of cross-linking and therefore higher flexibility. ${ }^{[32]}$ Furthermore, more positive oxidation potentials might also lead to a deeper degradation-cross-linking gradient in the thicker film obtained by electropolymerization at a more anodic $(0.8 \mathrm{~V})$ potential than the thinner one $(0.7 \mathrm{~V}) .{ }^{[33]}$ The characteristic response time to a change in polarity of the feeder electrodes is also faster in the case of films obtained at $0.7 \mathrm{~V}$. All these phenomena increase the ability to produce more pronounced crawling motion with the thinner film.
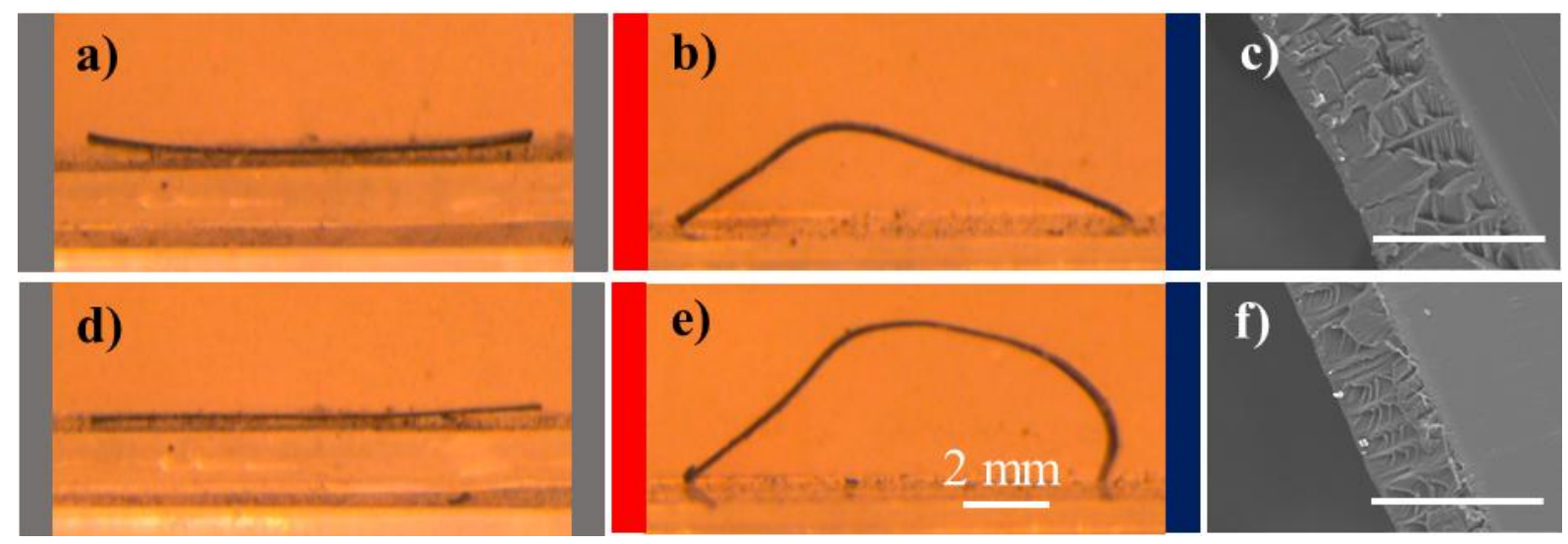
Figure 4 a) Thick polypyrrole film in the absence of electric field b) in the presence of electric field after the $8^{\text {th }}$ bipolar cycle and c) corresponding SEM image of the film section. d) Thin polypyrrole film in the absence of electric field e) in the presence of electric field after the $12^{\text {th }}$ bipolar cycle and $\mathrm{f}$ ) corresponding SEM image of its section $\left(0.5 \mathrm{M} \mathrm{LiClO}_{4}, 20 \mathrm{~V}\right)$. Red: positive feeder electrode; blue: negative feeder electrode; gray: no potential at the feeder electrodes. The scale bar in the SEM images is $100 \mu \mathrm{m}$.

When comparing the crawling behavior of both polymer strips upon potential switching it becomes obvious that the thinner film shows a more efficient motion. The fast switching and strong bending in the case of the thin film is responsible for the directional crawling illustrated in Figure 5. The polymer is travelling over a distance of $\sim 4 \mathrm{~mm}$ when switching the orientation of the electric field 25 times (see Supplementary information video S1). The speed of motion can be modulated by changing different parameters like salt concentration, thickness of the polymer, applied voltage and switching frequency. Therefore, it is rather difficult to make a direct correlation between the applied voltage and the final crawling velocity. 

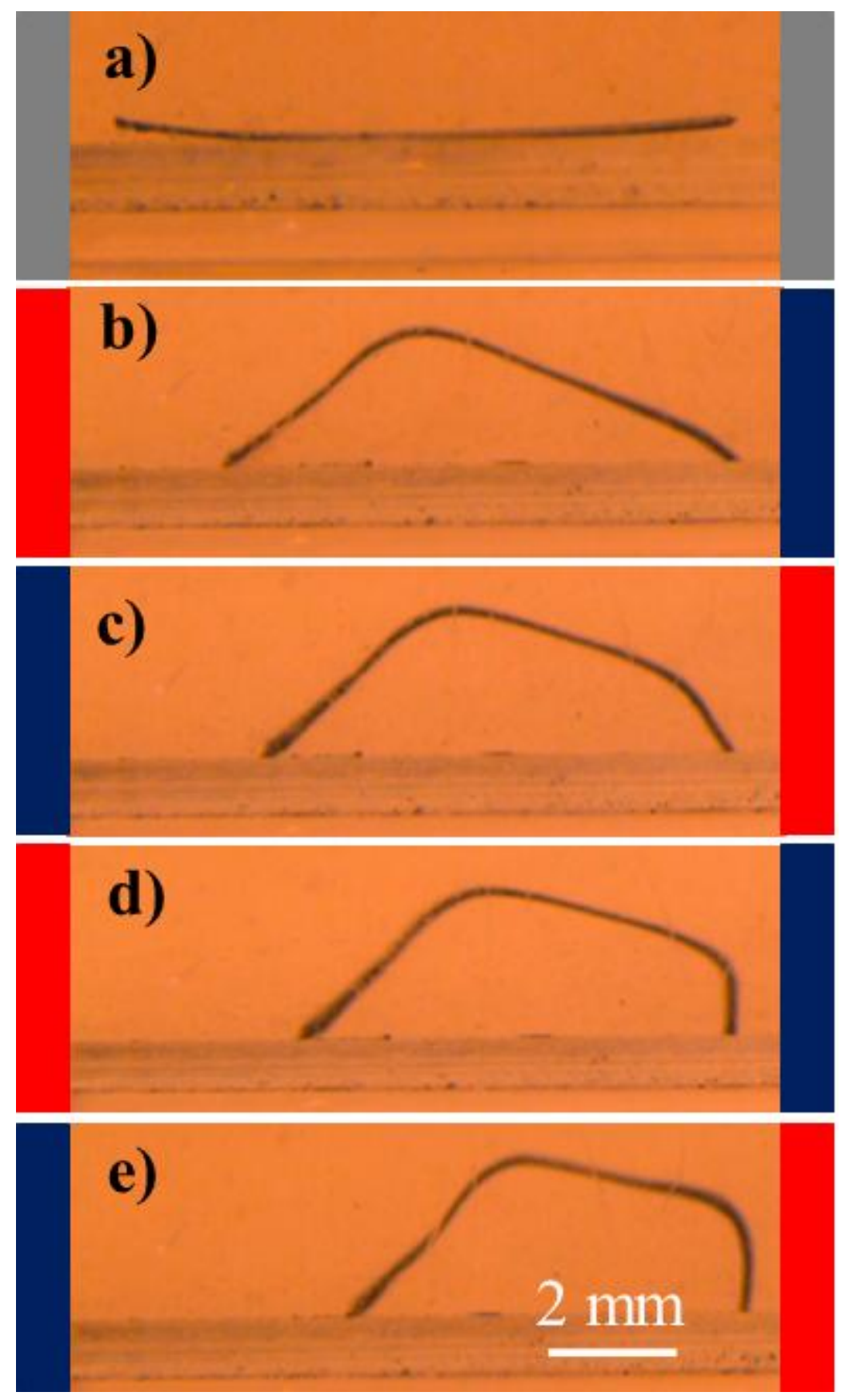

Figure 5: Different stages of polypyrrole film deformation during crawling motion a) before and b)-e) after a certain number of bipolar cycles at $20 \mathrm{~V}$ potential bias in $0.5 \mathrm{M} \mathrm{LiClO}_{4}$; b) $6^{\text {th }}$ c) $9^{\text {th }}$ d) $20^{\text {th }}$ and e) $25^{\text {th }}$ cycle.

\section{Conclusion}

We explored the possibility to use bipolar electrochemistry for triggering a crawling motion of a thin polypyrrole film. The synergetic combination of three different types of symmetry 
breaking is used to induce global locomotion of the object. An initial asymmetric surface roughness of the synthesized polymer allows preferential ion exchange on one side of the film, which in this way constitutes an intrinsic bilayer architecture. The second source of asymmetry is the polarization of the polymer object by the electric field present in solution. This allows carrying out a reduction reaction at one extremity of the film, whereas the opposite extremity undergoes an oxidation. Finally, the third break of symmetry originates from an irreversible electrochemical transformation of one end of the film due to its overoxidation during the first polarization cycle. This makes the two extremities distinguishable in terms of mechanical behavior in the subsequent polarization cycles and thus leads to directed crawling motion during reversible bending and relaxation. The main advantage of the here presented proof-of-concept experiments is that, in contrast to classic electrochemical actuation of conducting polymers, the movement can be induced in a wireless way. This, combined with a future downscaling of the object's size, opens very interesting perspectives in the frame of polymer based artificial muscles analogs with advanced functionalities.

\section{Experimental Section}

Synthesis of polypyrrole

Polypyrrole was synthesized by potentiostatic polymerization in aqueous medium following a literature protocol. ${ }^{[29]}$ A three electrode electrochemical cell (Reference electrode: Ag/AgCl, counter electrode: gold coated glass, working electrode: gold coated glass) was used for the polymerization. 0.2 M pyrrole (Sigma Aldrich) and 0.2 M sodium dodecylbenzene sulfonate (Sigma Aldrich) was dissolved in $10 \mathrm{ml}$ of distilled water. Parallel orientated gold coated electrodes $(1.2 \times 1.5 \mathrm{~cm})$ were kept at a distance of $2.5 \mathrm{~cm}$. For generating thin and thick polymer films a potential of 0.7 and $0.8 \mathrm{~V}$ was applied for polymerization during 2 hours, respectively. After completion of the polymerization process, the polymer was washed thoroughly with distilled water, dried under a $\mathrm{N}_{2}$ stream and finally peeled off the surface 
using a sharp blade. For the partial reduction of the polypyrrole film, the polymer was immersed in monomer free $0.1 \mathrm{M}$ sodium dodecylbenzenesulfonate solution and $-1 \mathrm{~V}$ was applied for 1 hour vs $\mathrm{Ag} / \mathrm{AgCl}$. After completion of the reduction process, the film was washed in water, dried and peeled off the working electrode with the help of a sharp blade. The as-synthesized or partially reduced polypyrrole films were used for the subsequent studies.

\section{Characterization}

The surface morphologies of the prepared films were characterized using a tabletop electron microscope (Hitachi, TM-1000) after Au sputtering on the polymer surface to reduce electric charging.

\section{Wireless bipolar motion}

Wireless bipolar motion was accomplished in a bipolar cell equipped with two graphite electrodes $(1 \mathrm{~cm}$ diameter) separated by a distance of $5 \mathrm{~cm}$. The rectangular polymer film (10 $\mathrm{x} 1 \mathrm{~mm}^{2}$ ) was kept in the center (laying at the bottom) of the bipolar cell filled with $0.5 \mathrm{M}$ $\mathrm{LiClO}_{4}$. A potential of $20 \mathrm{~V}$ was applied between the two graphite electrodes, and the polarity of the electrodes was switched every 5 seconds. To avoid parasitic effects of gas bubbles generated at the feeder electrodes, the center part of the bipolar cell containing the polymer strip is separated by two Nafion membranes. Phenomena of bending and generated locomotion were recorded with a macroscope (LEICA Z16 APO) in the video mode. Several dozens of polymer strips were characterized with respect to their dynamic behavior, and in all cases they behave similarly from an qualitative point of view. However, one can observe some variations in the efficiency of motion from one polymer strip to another, because thicknesses and defects are not necessarily completely identical.

\section{Acknowledgements}


This work was supported by the 2016 Post-Doctoral Fellowship Program of the Initiative of Excellence IdEx Bordeaux. A.K. acknowledges financial support from the Institut Universitaire de France. This project has also received funding from the European Research Council (ERC) under the European Union's Horizon 2020 research and innovation program (grant agreement $\mathrm{n}^{\circ}$ 741251, ERC Advanced grant ELECTRA).

\section{References}

[1] J. Katuri, X. Ma, M. M. Stanton, S. Sánchez, Acc. Chem. Res. 2017, 50, 2.

[2] G. Loget, A. Kuhn, J. Am. Chem. Soc. 2010, 132, 15918.

[3] W. Gao, X. Feng, A. Pei, C. R. Kane, R. Tam, C. Hennessy, J. Wang, Nano Lett. 2014, 14, 305.

[4] K. K. Dey, X. Zhao, B. M. Tansi, W. J. Méndez-Ortiz, U. M. Córdova-Figueroa, R. Golestanian, A. Sen, Nano Lett. 2015, 15, 8311.

[5] W. F. Paxton, K. C. Kistler, C. C. Olmeda, A. Sen, S. K. St. Angelo, Y. Cao, T. E. Mallouk, P. E. Lammert, V. H. Crespi, J. Am. Chem. Soc. 2004, 126, 13424;

[6] J. Roche, S. Carrara, J. Sanchez, J. Lannelongue, G. Loget, L. Bouffier, P. Fischer, A. Kuhn, Sci. Rep. 2014, 4, 6705.

[7] Y. Yoshizumi, H. Suzuki, ACS Appl. Mater. Interfaces 2017, 9, 21355.

[8] S. Campuzano, J. Orozco, D. Kagan, M. Guix, W. Gao, S. Sattayasamitsathit, J. C. Claussen, A. Merkoçi, J. Wang, Nano Lett. 2012, 12, 396.

[9] W. Wang, L. A. Castro, M. Hoyos, T. E. Mallouk, ACS Nano 2012, 6, 6122.

[10] Y. Yoshizumi, K. Okubo, M. Yokokawa, H. Suzuki, Langmuir 2016, 32, 9381.

[11] Y. Wang, R. M. Hernandez, D. J. Bartlett, J. M. Bingham, T. R. Kline, A. Sen, T. E. Mallouk, Langmuir 2006, 22, 10451.

[12] P. Degen, Curr. Opin. Colloid Interface Sci. 2014, 19, 611.

[13] L. Bouffier, V. Ravaine, N. Sojic, A. Kuhn, Curr. Opin. Colloid Interface Sci. 2016, $21,57$.

[14] J.-Z. Jiang, M.-H. Guo, F.-Z. Yao, J. Li, J.-J. Sun, RSC Adv. 2017, 7, 6297.

[15] S. Palagi, A. G. Mark, S. Y. Reigh, K. Melde, T. Qiu, H. Zeng, C. Parmeggiani, D. Martella, A. Sanchez-Castillo, N. Kapernaum, F. Giesselmann, D. S. Wiersma, E. Lauga, P. Fischer, Nat. Mater. 2016, 15, 647.

[16] E. Diller, J. Zhuang, G. Zhan Lum, M. R. Edwards, M. Sitti, Appl. Phys. Lett. 2014, 104, 174101.

[17] M. Hoop, Y. Shen, X.-Z. Chen, F. Mushtaq, L. M. Iuliano, M. S. Sakar, A. Petruska, M. J. Loessner, B. J. Nelson, S. Pané, Adv. Funct. Mater. 2016, 26, 1063.

[18] G. Loget, D. Zigah, L. Bouffier, N. Sojic, A. Kuhn, Acc. Chem. Res. 2013, 46, 2513.

[19] L. Bouffiar, D. Zigah, N. Sojic, A. Kuhn, in Electroanalytical Chemistry: A Series of Advances, Vol. 27 (Ed: C. G. Zoski, A. J. Bard), CRC Press 2017, Ch. 2.

[20] S. E. Fosdick, K. N. Knust, K. Scida, R. M. Crooks, Angew. Chem. Int. Ed. 2013, 52, 10438.

[21] L. Koefoed, S. U. Pedersen, K. Daasbjerg, Curr. Opin. Electrochem. 2017, 2, 13.

[22] B. Gupta, B. Goudeau, A. Kuhn, Angew. Chem. Int. Ed., 2017, DOI: 10.1002/anie.201709038n/a.

[23] R. H. Baughman, Synth. Met. 1996, 78, 339.

[24] T. F. Otero, J. M. Sansiñena, Bioelectrochem. Bioenerg. 1995, 38, 411.

[25] L. Bay, K. West, P. Sommer-Larsen, S. Skaarup, M. Benslimane, Adv. Mater. 2003, 15, 310;

[26] R. Balint, N. J. Cassidy, S. H. Cartmell, Acta Biomater. 2014, 10, 2341. 
[27] A. Gursel, D. Valerie, R. Philippe, S. Geoff, J. Micromech. Microeng. 2009, 19, 025017.

[28] E. W. H. Jager, O. Inganäs, I. Lundström, Science 2000, 288, 2335.

[29] F. Garcia-Cordova, L. Valero, Y. A. Ismail, T. F. Otero, J. Mater. Chem. 2011, 21, 17265.

[30] I. Mehmet, S. Mohammadreza, A. Gursel, Smart Mater. Struct. 2014, 23, 125024.

[31] S. D. Deshpande, K. Jaehwan, Y. Sung-Ryul, Smart Mater. Struct. 2005, 14, 876.

[32] C. C. Bof Bufon, J. Vollmer, T. Heinzel, P. Espindola, H. John, J. Heinze, J. Phys. Chem. B 2005, 109, 19191.

[33] T. F. Otero Polym. Rev. 2013, 53, 311. 